\title{
Substrate impact on the low-temperature growth of GaN thin films by plasma-assisted atomic layer deposition
}

Seda Kizir, Ali Haider, and Necmi Biyikli

Citation: Journal of Vacuum Science \& Technology A 34, 041511 (2016); doi: 10.1116/1.4953463

View online: $\mathrm{https}: / /$ doi.org/10.1116/1.4953463

View Table of Contents: http://avs.scitation.org/toc/jva/34/4

Published by the American Vacuum Society

\section{Articles you may be interested in}

Substrate temperature influence on the properties of GaN thin films grown by hollow-cathode plasma-assisted atomic layer deposition

Journal of Vacuum Science \& Technology A: Vacuum, Surfaces, and Films 34, 01 A125 (2016);

$10.1116 / 1.4936230$

Atomic layer deposition of $\mathrm{GaN}$ at low temperatures

Journal of Vacuum Science \& Technology A: Vacuum, Surfaces, and Films 30, 01A124 (2012);

$10.1116 / 1.3664102$

Comparison of trimethylgallium and triethylgallium as "Ga" source materials for the growth of ultrathin GaN films on $\mathrm{Si}(100)$ substrates via hollow-cathode plasma-assisted atomic layer deposition

Journal of Vacuum Science \& Technology A: Vacuum, Surfaces, and Films 34, 01 A137 (2016);

$10.1116 / 1.4937725$

Demonstration of flexible thin film transistors with GaN channels

Applied Physics Letters 109, 233504 (2016); 10.1063/1.4971837

Low-temperature self-limiting atomic layer deposition of wurtzite InN on $\mathrm{Si}(100)$

AIP Advances 6, 045203 (2016); 10.1063/1.4946786

Low temperature thin film transistors with hollow cathode plasma-assisted atomic layer deposition based GaN channels

Applied Physics Letters 104, 243505 (2014); 10.1063/1.4884061

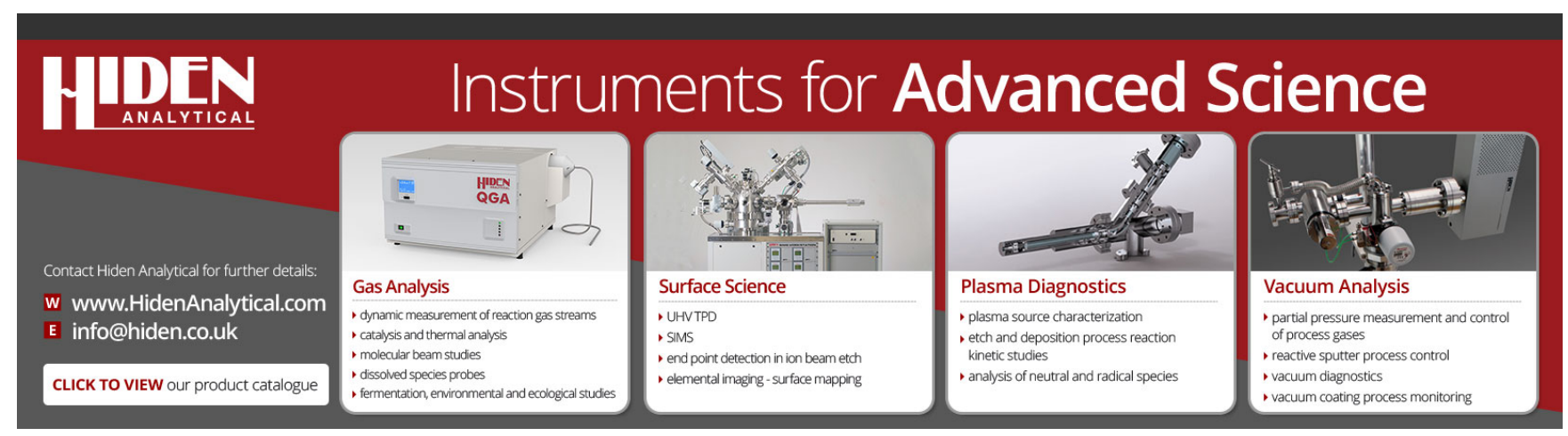




\title{
Substrate impact on the low-temperature growth of GaN thin films by plasma-assisted atomic layer deposition
}

\author{
Seda Kizir, Ali Haider, and Necmi Biyiklia) \\ National Nanotechnology Research Center (UNAM), Bilkent University, Bilkent, Ankara 06800, Turkey \\ and Institute of Materials Science and Nanotechnology, Bilkent University, Bilkent, Ankara 06800, Turkey
}

(Received 8 January 2016; accepted 24 May 2016; published 13 June 2016)

Gallium nitride $(\mathrm{GaN})$ thin films were grown on $\mathrm{Si}(100), \mathrm{Si}(111)$, and c-plane sapphire substrates at $200^{\circ} \mathrm{C}$ via hollow-cathode plasma-assisted atomic layer deposition (HCPA-ALD) using $\mathrm{GaEt}_{3}$ and $\mathrm{N}_{2} / \mathrm{H}_{2}$ plasma as group-III and $\mathrm{V}$ precursors, respectively. The main aim of the study was to investigate the impact of substrate on the material properties of low-temperature ALD-grown GaN layers. Structural, chemical, and optical characterizations were carried out in order to evaluate and compare film quality of GaN on different substrates. X-ray reflectivity measurements showed film density values of $5.70,5.74$, and $5.54 \mathrm{~g} / \mathrm{cm}^{3}$ for GaN grown on $\mathrm{Si}(100)$, $\mathrm{Si}$ (111), and sapphire, respectively. Grazing incidence x-ray diffraction measurements exhibited hexagonal wurtzite structure in all HCPA-ALD grown GaN samples. However, dominant diffraction peak for GaN films grown on Si and sapphire substrates were detected differently as (002) and (103), respectively. Xray diffraction gonio scans measured from $\mathrm{GaN}$ grown on c-plane sapphire primarily showed (002) orientation. All samples exhibited similar refractive index values $(\sim 2.17$ at $632 \mathrm{~nm})$ with $2-3$ at. $\%$ of oxygen impurity existing within the bulk of the films. The grain size was calculated as $\sim 9-10 \mathrm{~nm}$ for GaN grown on $\mathrm{Si}(100)$ and $\mathrm{Si}$ (111) samples while it was $\sim 5 \mathrm{~nm}$ for GaN/sapphire sample. Root-mean-square surface roughness values found as $0.68,0.76$, and $1.83 \mathrm{~nm}$ for GaN deposited on Si (100), Si (111), and sapphire, respectively. Another significant difference observed between the samples was the film growth per cycle: GaN/sapphire sample showed a considerable higher thickness value when compared with $\mathrm{GaN} / \mathrm{Si}$ samples, which might be attributed to a possibly more-efficient nitridation and faster nucleation of sapphire surface. (C) 2016 American Vacuum Society. [http://dx.doi.org/10.1116/1.4953463]

\section{INTRODUCTION}

Featuring a direct and wide band gap, gallium nitride $(\mathrm{GaN})$ manifest solid solutions with both aluminum nitride (AIN) and indium nitride ( $\mathrm{InN})$, providing energy band gap values which can be tuned from the ultraviolet to infrared range. ${ }^{1-5}$ GaN-based alloys became the materials of choice for solid state lighting with InGaN/GaN-based blue light emitting diodes, AlGaN/GaN-based visible and solar-blind UVphotodetectors, and high-performance blue laser diodes. ${ }^{6-8}$ Moreover, state-of-the-art RF high-power electronics rely heavily on durable $\mathrm{AlGaN} / \mathrm{GaN}$-based high-electron mobility transistors, exhibiting excellent high-frequency and highpower handling performance., ${ }^{9,10}$

Single crystal GaN epilayers with relatively low defect density have been grown most widely on sapphire substrates using high-temperature methods such as metal organic chemical vapor deposition (MOCVD) ${ }^{11-13}$ and molecular beam epitaxy. ${ }^{14}$ However, there are two main causes which make sapphire a nonideal substrate for epitaxy of $\mathrm{GaN}$ in electronic devices: a rather large mismatch of the substrate lattices $(\sim 16 \%)$ and significantly higher thermal expansion coefficient as compared to GaN [thermal expansion mismatch (a): $-34.7 \%] .^{15,16}$ Large lattice mismatch yields high structural defect density, while difference in thermal expansion coefficient typically results in highly stressed films

${ }^{\text {a)} E l e c t r o n i c ~ m a i l: ~ b i y i k l i @ u n a m . b i l k e n t . e d u . t r ~}$ which might lead to additional structural misfits or even cracking. Such high defect density levels significantly decrease the mobility of charged carriers and life time of minority carriers. Furthermore, due to large thermal expansion coefficient difference, biaxial compressive strain arises within the epilayer after cooling. An attractive alternative substrate of interest is silicon ( $\mathrm{Si}$ ) with a lattice mismatch of $\sim 17 \%$ for $\langle 111\rangle$ orientation. Si has superior crystal quality, excellent doping properties, low materials cost, as well as default compatibility with micro/nanoscale device fabrication equipment. ${ }^{17}$ However, even higher lattice and thermal expansion coefficient mismatch between $\mathrm{GaN}$ and Si hinders the production of high-quality defect-free $\mathrm{GaN}$ films on $\mathrm{Si}$ substrates [thermal expansion mismatch (a): $-25.3 \%$, $-115 \%$ ]. ${ }^{16}$ These two important limitations have been solved to a certain extent by introducing certain buffer layers ( $\mathrm{AlN}, \mathrm{AlGaN}$, or $\mathrm{GaN}$ ) between the substrate and $\mathrm{GaN}$ device layer in order to reduce the defect density. ${ }^{15,18,19}$

Choice and preparation of substrates is a very delicate issue for heteroepitaxy of $\mathrm{GaN}$, as the substrate influences crystal orientation, surface morphology, polarity, strain, and defect concentration of the over-grown films at a considerable extent. Therefore, it is captivating to investigate the effect of major substrate materials on GaN layers deposited by alternative growth techniques which might be used for post-CMOS processing toward III-nitride/Si integration. Atomic layer deposition (ALD) is such a low temperature deposition technique which is suitable for growing materials 
with excellent uniformity and ultimate conformality. Growth mechanism of ALD is based on surface reactions which occur at the available sites of the surface and show selfterminating character after all available chemisorption sites are occupied. This "self-terminating" behavior of ALD allows to control the film thickness at sub-Angstrom level. Plasma assisted ALD (PA-ALD) is a modified form of ALD in which energetic radicals assist in accelerating the selflimiting ALD reactions. PA-ALD offers additional merits over thermal ALD such as reduced growth temperatures and improved material properties. ${ }^{20,21}$

In the initial reports of ALD-grown GaN films, film growth was achieved at higher temperatures $\left(500-750^{\circ} \mathrm{C}\right)$ by using $\mathrm{GaCl}_{3}$ and $\mathrm{NH}_{3}$ precursor materials. ${ }^{22}$ Follow up work for low-temperature (as low as $200^{\circ} \mathrm{C}$ ) growth of crystalline GaN using PA-ALD has been successfully demonstrated by several groups utilizing $\mathrm{GaMe}_{3}$ or $\mathrm{GaEt}_{3}$ in conjunction with $\mathrm{N}_{2} / \mathrm{H}_{2}$ or $\mathrm{NH}_{3}$ plasma as $\mathrm{Ga}$ and nitrogen precursor sources, respectively. ${ }^{5,23-26}$ However, a comparative study investigating the influence of different substrates on the material properties of $\mathrm{GaN}$ grown using lowtemperature PA-ALD is lacking. Recently, the effect of different substrates on material properties of PA-ALD grown AlN thin films has been investigated, where they demonstrate that $\mathrm{Si}(111)$ is a better substrate for AlN growth. ${ }^{27} \mathrm{In}$ this work, we have studied and compared the properties of $\mathrm{GaN}$ thin films grown on $\mathrm{Si}$ (100), $\mathrm{Si}$ (111), and c-plane sapphire substrates by using hollow-cathode plasma-assisted ALD (HCPA-ALD) at $200^{\circ} \mathrm{C}$.

\section{EXPERIMENT}

\section{A. Film growth}

GaN thin film deposition is performed in Fiji F200-LL ALD reactor (Ultratech/CambridgeNanotech, Inc., Cambridge, MA) with a modified configuration of stainless-steel hollowcathode plasma source (Meaglow, Ltd., Canada). Hollow cathode plasma (HCP) source has been found superior to quartz-based inductively coupled plasma source as our former studies on ALD-grown GaN confirmed a significantly higher layer quality with considerably lower impurity incorporation. ${ }^{5}$ Moreover, HCP source possesses advantages such as high plasma density and rapid on/off cycles for pulsed growth..$^{28,29}$ Prior to growth experiments, $\mathrm{Si}$ (111), Si (100), and sapphire substrates were cleaned sequentially using isopropanol, acetone, methanol, and deionized water in an ultrasonic bath. In order to remove the native oxide layer on the substrate surface, additional diluted HF (2\%) cleaning process was applied for 2 $\mathrm{min}$. Afterward, samples were loaded immediately to the reactor, and the process base pressure was adjusted to $150 \mathrm{mTorr}$. To prepare an enhanced nitridated surface in order to eliminate possible nucleation delays and to enhance the crystalline quality, a nitridation process step was utilized before the ALD cycles by applying $60 \mathrm{~s}$ of $\mathrm{N}_{2} / \mathrm{H}_{2}$ plasma and subsequently 60 $\mathrm{s}$ of $\mathrm{N}_{2}$ plasma. ${ }^{30} \mathrm{GaEt}_{3}$ was used as organometallic precursor for $\mathrm{Ga}$ with a pulse length of $0.5 \mathrm{~s}$. As nitrogen half-cycle, $\mathrm{N}_{2} / \mathrm{H}_{2}$ plasma exposure is applied with $50 / 50 \mathrm{sccm}$ flow rate for $60 \mathrm{~s}$ under $300 \mathrm{~W}$ rf-plasma power. Ten seconds purge intervals were used in between the metal-organic precursor pulse and $\mathrm{N}_{2} / \mathrm{H}_{2}$ plasma exposures to evacuate the excess precursor molecules and reaction byproducts. Ar is used as the carrier and plasma gas with 30 and $100 \mathrm{sscm}$ flow rates for $\mathrm{GaEt}_{3}$ and $\mathrm{N}_{2} / \mathrm{H}_{2}$ flows, respectively. A total of 1000 growth cycles were carried out at a substrate temperature of $200^{\circ} \mathrm{C}$.

\section{B. Film characterization}

Both x-ray reflectivity (XRR) and grazing-incidence x-ray diffraction (GIXRD) measurements were performed with PANalytical X'Pert PRO MRD diffractometer (PANalytical B. V., Netherlands) by using $\mathrm{Cu} \mathrm{K}_{\alpha}$ radiation. GIXRD spectra are collected at $0.1^{\circ}$ step size and $10 \mathrm{~s}$ of counting time in the $2 \Theta$ range of $20^{\circ}-80^{\circ}$. Omega- $2 \Theta$ scan is performed from $0^{\circ}$ to $2.5^{\circ}$ for XRR measurements. X'pert pro MPD (PANalytical, Netherlands) system is used for X-ray diffraction (XRD) measurements with Bragg Brentano geometry. Gonio scan has been performed with a step size of $0.0016^{\circ}$ in between $33.5^{\circ}$ and $35^{\circ}$ using a counting time of $10 \mathrm{~s}$. Interplanar spacing $\left(d_{h k l}\right)$ values were evaluated from peak positions using the well-known Bragg's law. Lattice parameter $c$ was calculated by substituting $d_{h k l}$ values in Eq. (1), which relates the interplanar spacing $\left(d_{h k l}\right)$, miller indices $(h k l)$, and lattice parameters ( $a$ and $c$ ) for hexagonal crystals

$$
\frac{1}{d^{2}}=\frac{4}{3}\left(\frac{h^{2}+h k+k^{2}}{a^{2}}\right)+\frac{l^{2}}{c^{2}} .
$$

Ellipsometric measurements were recorded in 300$1000 \mathrm{~nm}$ wavelength interval for three different angles of incidence $\left(65^{\circ}-70^{\circ}-75^{\circ}\right)$ by variable-angle spectroscopic ellipsometer (V-VASE, J. A. Woollam Co., Inc., NE) and a rotating analyzer. Optical constants and film thicknesses of the samples were extracted from the measured ellipsometer data by applying a fitting procedure (WVASE 32 software) with Cauchy dispersion function and Tauc Lorentz oscillator. The absorption coefficient

$$
\alpha(\lambda)=\frac{4 \pi k(\lambda)}{\lambda}
$$

was calculated from $k(\lambda)$ values determined from the ellipsometry data. Optical band gap $\left(E_{g}\right)$ is expressed by the following equation for direct band gap materials, ${ }^{31}$ which can be analytically extracted via extrapolation of the linear part of the absorption spectrum to $(\alpha E)^{2}=0$

$$
\alpha E=A\left(E-E_{g}\right)^{1 / 2} .
$$

In order to determine the elemental composition and chemical bonding states of the film, x-ray photoelectron spectroscopy was implemented using a Thermo Scientific KAlpha spectrometer (Thermo Fisher Scientific, MA) with monochromatized $\mathrm{Al} \mathrm{K}_{\alpha}$ source. For depth profiling, GaN thin films were etched with an energy and spot size of $1 \mathrm{kV}$ and $400 \mu \mathrm{m}$ Ar beam, respectively. Surface morphologies of the $\mathrm{GaN}$ thin films were analyzed using high-resolution 
scanning electron microscopy (HR-SEM, FEI, Hillsboro, OR NNL 600i), and atomic force microscopy (Asylum Research MFP-3D) was operated in tapping mode.

\section{RESULTS AND DISCUSSION}

\section{A. Structural and optical characterizations}

XRR measurements showed characteristic Kiessig fringes of three GaN samples grown on different substrates in Fig. 1(a). Periodicity and amplitude of oscillations provides information about film thickness and film density, respectively. In order to extract this information, X-pert reflectivity software was used to fit the experimental data by using four and three-layer models, i.e., $\mathrm{Ga}_{2} \mathrm{O}_{3} / \mathrm{GaN} / \mathrm{SiO}_{2} / \mathrm{Si}$ and $\mathrm{Ga}_{2} \mathrm{O}_{3} /$ $\mathrm{GaN} / \mathrm{Al}_{2} \mathrm{O}_{3}$ for samples grown on $\mathrm{Si}$ and sapphire substrates, respectively. Figure 1 (b) shows the measured versus simulated XRR data for $\mathrm{GaN} / \mathrm{Si}(111)$ sample, confirming the close agreement between experimental and calculated data.

$\mathrm{X}$-rays, when impinged on a flat surface with small (grazing) angle, undergo total reflection below a critical angle $\left(\Theta_{c}\right)$. X-ray intensity drops abruptly after exceeding the critical angle as shown for $\mathrm{GaN} / \mathrm{Si}(111), \mathrm{GaN} / \mathrm{Si}(100)$, and $\mathrm{GaN} /$ sapphire, as shown in Fig. 1(c). Critical angle is a specific material property depending primarily on the film density. When the second derivative of the XRR intensity $d^{2} I / d^{2} \Theta$ is plotted versus $\Theta$ [Fig. 1(d)], threshold of X-ray penetration is located at the first minima of this curve. For GaN/Si (100) and $\mathrm{GaN} / \mathrm{sapphire}$ samples, the critical angle was extracted as $\sim 0.32^{\circ}$, while for $\mathrm{GaN} / \mathrm{Si}(111)$ sample, this value is found as $\sim 0.33^{\circ}$. Motamedi et $a l .{ }^{24}$ recently reported very similar values in their GaN thin films grown by PA-ALD revealing critical angles of $0.32^{\circ}$, while this value is around $0.36^{\circ}$ for single crystalline bulk GaN. Density values extracted from simulations reveal that $\mathrm{GaN} / \mathrm{Si}(111)$ exhibit the highest density value of $5.74 \mathrm{~g} / \mathrm{cm}^{3}$, while $\mathrm{GaN}$ deposited on $\mathrm{Si}(100)$ and sapphire possesses density values of 5.70 and $5.54 \mathrm{~g} /$ $\mathrm{cm}^{3}$, respectively. The density value of GaN/Si (111) is relatively closer to the bulk GaN density value of $6.15 \mathrm{~g} / \mathrm{cm}^{3}$. For extracting the thickness of $\mathrm{GaN}$ films, a $\mathrm{Ga}_{2} \mathrm{O}_{3}$ layer was added on top of GaN in XRR fitting software and thickness values of two layers were summed-up $\left(d_{\mathrm{GaN}}+d_{\mathrm{Ga}_{2} \mathrm{O}_{3}}\right)$ to obtain final thickness of the films. Thickness values came out as $\sim 29, \sim 32$, and $\sim 34 \mathrm{~nm}$ for GaN deposited on $\mathrm{Si}$ (111), Si (100), and sapphire substrates, respectively. In addition, interlayer roughness values in between $\mathrm{GaN}$ and $\mathrm{Ga}_{2} \mathrm{O}_{3}$ layers was found as $1.1,2.4$, and $5.3 \mathrm{~nm}$ for $\mathrm{GaN}$ deposited on the same sequence of substrates. Higher interlayer roughness obtained from $\mathrm{GaN}$ grown on sapphire led to considerably higher fit parameters for data fitting which implies that thickness and density estimation of GaN grown on sapphire cannot be trusted with absolute certainty.

Optical properties, including refractive index dispersion curves and optical band gap, were determined using spectroscopic ellipsometric measurements and related data analysis. Figure 2(a) shows the comparison of refractive index values of GaN films deposited on different substrates. The refractive index values at $632 \mathrm{~nm}$ of $\mathrm{GaN}$ on $\mathrm{Si}$ (111), $\mathrm{Si}$ (100), and sapphire were measured as 2.16, 2.17, and 2.18, respectively. Refractive index value around 2.4 is reported for single crystal hexagonal wurtzite GaN, which is notably higher than the values obtained in our films. ${ }^{32}$ In the case of polycrystalline hexagonal wurtzite $\mathrm{GaN}$ thin films, refractive
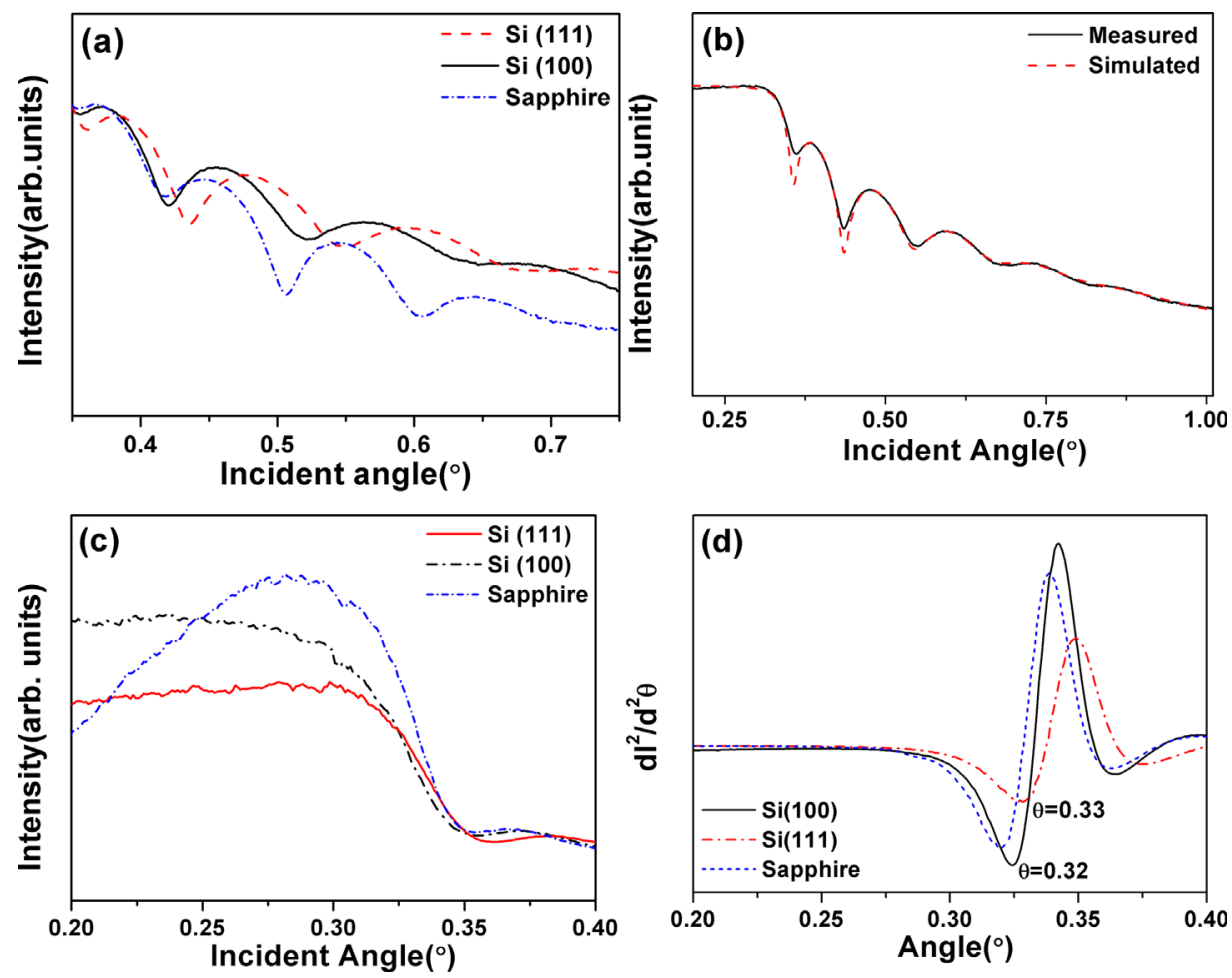

FIG. 1. (Color online) XRR results for GaN thin films grown on Si (111), Si (100), and sapphire substrates; (a) characteristic Kiessig fringes, (b) measured vs simulated data for GaN/Si(111), (c) measured angular dependence around critical angle, and (d) critical angle extraction. 

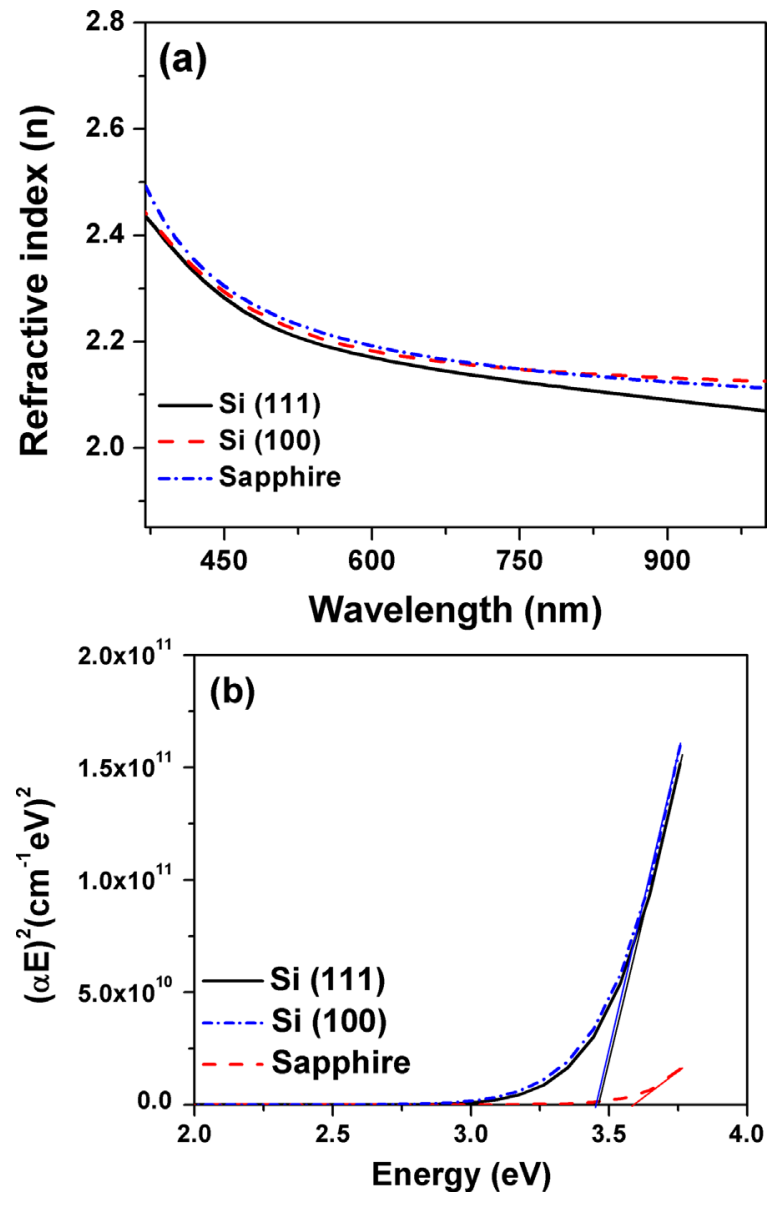

FIG. 2. (Color online) (a) Spectral refractive index values for GaN/Si(111), $\mathrm{GaN} / \mathrm{Si}(100)$, and $\mathrm{GaN} /$ sapphire samples. (b) Calculated optical band gap values of $\mathrm{GaN}$ grown on different substrates.

index values between 2.10 and 2.17 were reported in literature, which resembles the data obtained in this study., Spectroscopic ellipsometer data fitting reveals thickness of GaN grown on $\mathrm{Si}$ (100), $\mathrm{Si}$ (111), and sapphire as 33, 35, and $43 \mathrm{~nm}$, respectively. These thickness values correspond to growth per cycle (GPC) values of $0.33,0.35$, and $0.43 \AA$ / cycle for $\mathrm{GaN}$ grown on same sequence of substrates. As stated in Sec. II, Si substrates were treated with HF prior to GaN growth, and the ellipsometric spectra were fitted using a two-layer model [i.e., Cauchy/Si $(0.5 \mathrm{~mm})]$. It was assumed that the thickness of oxide layer on $\mathrm{Si}$ is negligible at $\mathrm{GaN} / \mathrm{Si}$ interface for ellipsometric data fittings. On the other hand, when performing XRR fitting, $\mathrm{SiO}_{2}$ layer was taken into account, and data were fitted using a four-layer model (i.e., $\mathrm{Ga}_{2} \mathrm{O}_{3} / \mathrm{GaN} / \mathrm{SiO}_{2} / \mathrm{Si}$ ). Since a default $\mathrm{Ga}_{2} \mathrm{O}_{3}$ layer is not defined in the database of the ellipsometer wVASE 32 software, we have used the conventional Cauchy model to determine the layer thickness. The different models used in two different measurement techniques (XRR and ellipsometry) might have led to the non-negligible difference in estimated film thickness.

It is reported in the literature that the initial nitridation process using $\mathrm{N}_{2}$ plasma species induces the formation of a thin AlN layer on the surface of sapphire. ${ }^{23}$ Higher film thickness values extracted in the case of $\mathrm{GaN}$ grown on sapphire might be the result of a more-efficient and faster nucleation of $\mathrm{GaN}$ on this AlN monolayer. This implies that GPC was most probably higher in the initial phase of $\mathrm{GaN}$ growth on sapphire and then it might have reduced to a lower steady state value. Optical band gap values were calculated from $(\alpha h \nu)^{2}$ vs $h \nu$ plot of the films. Extrapolating the straight line segment of the plot to abscissa provides the value of $\mathrm{E}_{\mathrm{g}}$ value, which came out to be $3.44,3.45$, and $3.61 \mathrm{eV}$ for GaN grown on $\mathrm{Si}$ (100), Si (111), and sapphire [Fig. 2(b)]. The optical band gap values of GaN grown on both $\mathrm{Si}$ substrates are reasonably close to the bulk $\mathrm{GaN}$ value $(\sim 3.4 \mathrm{eV})$. On the other hand, GaN/sapphire sample features a higher optical band edge which might originate from the smaller grain/crystallite structure to be discussed later in this section.

In order to identify the crystalline nature of the GaN thin films, GIXRD measurements were performed. GIXRD measurements revealed single-phase hexagonal wurtzite polycrystalline character of $\mathrm{GaN}$ irrespective of the substrate utilized (Fig. 3). Hexagonal wurtzite reflections of (010), (002), (110), (103), and (112) were manifested by GIXRD patterns of $\mathrm{GaN}$ grown on $\mathrm{Si}$ substrates. No other phase mixing was detected (i.e., cubic). In GIXRD, sample is exposed to x-rays at grazing angle of incidence in order to avoid intense signal from substrate and obtain a stronger signal from the thin film. ${ }^{33}$ In contrast to $\theta-2 \theta$ scans, GIXRD detects the tilted planes which are present in polycrystalline materials. However, by its inherent configuration, GIXRD is rather insensitive to planes which are parallel to the substrate surface. Although displaying quite similar diffraction patterns, $\mathrm{GaN} / \mathrm{Si}$ samples show a considerable difference in the peak intensity values: (002) peak of $\mathrm{GaN} / \mathrm{Si}(111)$ appeared to be higher compared to $\mathrm{GaN} / \mathrm{Si}(100)$. Full width at half maximum (FWHM) values obtained from (002) reflection of GaN film deposited on $\mathrm{Si}(111)$ and $\mathrm{Si}(100)$ was measured as 2484 and 2808 arc sec, respectively. On the other hand, (010), (110), and (112) reflections disappeared from GIXRD pattern of the GaN film deposited on c-plane sapphire substrate, which might be due to insufficient penetration of $\mathrm{x}$-rays inside the GaN layer grown on sapphire. The intensities of (100) and (002) reflections drastically decreased while the intensity of (103) refection showed a significant increase. XRD gonio scans have been conducted on $\mathrm{GaN}$ grown on $\mathrm{Si}$ (111), Si (100), and c-plane sapphire. However, due to low thickness of the grown films, strong background signal from $\mathrm{Si}$ does not allow us to determine the bulk orientation of these films. However, we were able to obtain the gonio-scan of GaN/sapphire samples. Regular powder diffraction setup (X'pert pro multipurpose diffractometer) is used for obtaining gonio (Bragg Brentano geometry) scans from GaN/sapphire sample, which primarily showed (002) orientation with a FWHM of 17.34 arc min [Fig. 3(b)] which is even narrower than the previously reported values for the PA-ALDgrown $\mathrm{GaN}$ on sapphire substrates. ${ }^{24}$

$\mathrm{GaN}$ directly grown (without any buffer layers) on $\mathrm{Si}$ at higher temperatures shows poor crystalline quality in the presence of a mixture of cubic and hexagonal phases. Si has a tendency to form an amorphous $\mathrm{Si}_{\mathrm{x}} \mathrm{N}_{\mathrm{y}}$ layer during the 

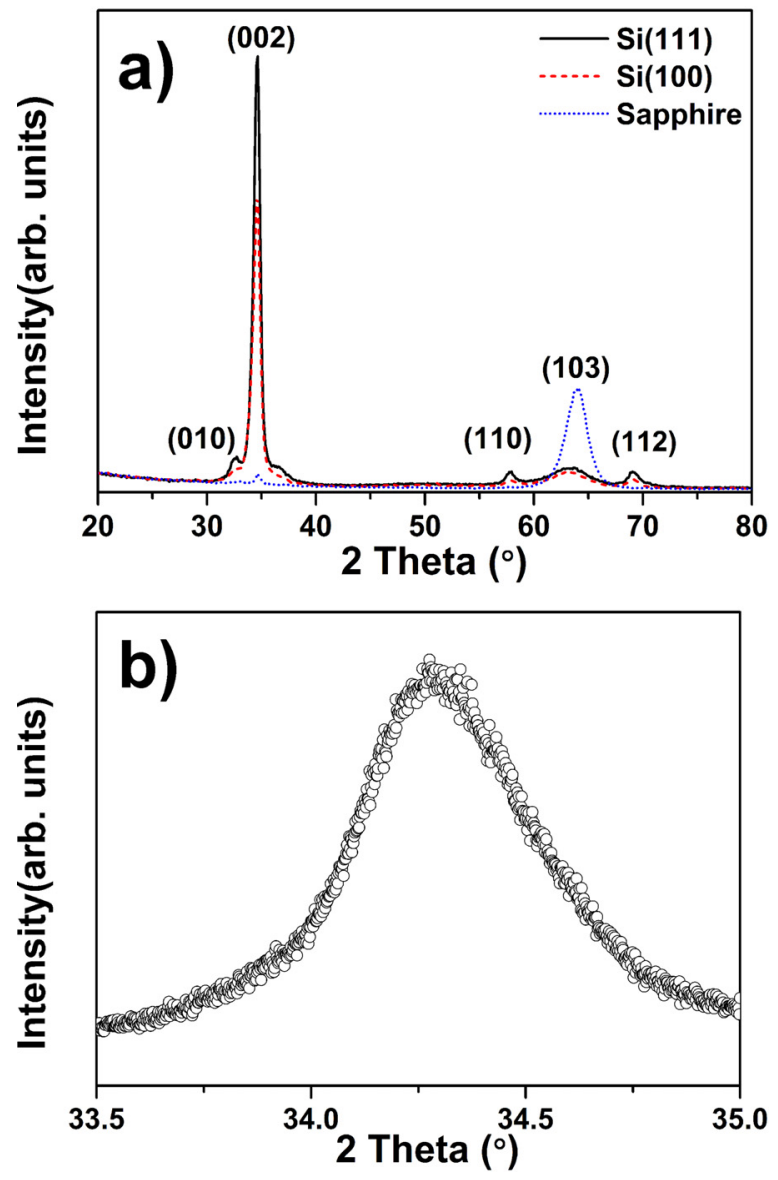

FIG. 3. (Color online) (a) GIXRD patterns of $\mathrm{GaN}$ thin films grown on $\mathrm{Si}$ (111), Si (100), and sapphire substrates; (b) XRD gonio scan of GaN/sapphire sample.

nucleation steps which gives rise to poor crystalline quality of GaN epilayers grown at higher temperatures. ${ }^{15,17}$ That effect was mitigated by introducing a thin $\mathrm{SiC}$ or AlN layer on $\mathrm{Si}$ substrates before the growth of GaN. In the case of lowtemperature $\mathrm{GaN}$ growth, $\mathrm{Si}_{\mathrm{x}} \mathrm{N}_{\mathrm{y}}$ layer formation might not be the prevailing factor to influence the crystalline quality due to the polycrystalline film character with relatively small crystallite sizes. Also, difference in the thermal expansion coefficient between substrate material and GaN would not affect the film properties significantly as growth takes place at substantially reduced temperatures, $200^{\circ} \mathrm{C}$ in the present case of HCPAALD. Apparently, improved crystalline quality and higher film density in the case of GaN grown on $\mathrm{Si}$ (111) might be due to the hexagonal nature of the specific $\mathrm{Si}$ (111) plane. In the literature, higher crystalline quality of the PE-ALD grown AlN was observed on $\mathrm{Si}$ (111), and it was mainly attributed to the hexagonal structure of $\mathrm{Si}(111)$ substrate. ${ }^{27}$

The lattice parameter $c$ was determined using the (002) reflection of hexagonal phase from diffraction patterns of $\mathrm{GaN}$ grown on different substrates. First interplanar spacing values $\left(d_{h k l}\right)$ were calculated using Bragg's law and these values were then substituted in Eq. (1), which provides a relationship between interplanar spacing $\left(d_{h k l}\right)$, miller indices, and lattice parameters for hexagonal crystals. The $c$-axis lattice parameter was found as the following: " $c_{\mathrm{GaN} / \mathrm{Si}(111)}=5.183 \AA$," " $c_{\mathrm{GaN} / \mathrm{Si}(100)}=5.185 \AA$," and " $c_{\mathrm{GaN} / \text { sapphire }}=5.175 \AA$." $C$ axis lattice parameter values of $\mathrm{GaN} / \mathrm{Si}$ samples are in good agreement with the bulk GaN value $(c=5.185 \AA) .{ }^{34}$ However, the variation in the $c$ axis lattice parameter of $\mathrm{GaN} / \mathrm{sapphire}$ with respect to their nominally strain-free counterpart possibly indicates the presence of a minor strain component in the PA-ALD-grown films.

HR-SEM measurements were conducted to observe the surface morphology and determine the grain sizes of GaN grown on $\mathrm{Si}$ (100), Si (111), and sapphire. Figure 4 shows the surface morphology of GaN grown on different substrates. Average size of polycrystalline grains was calculated using IMAGE J (image analysis software). The average grain size was calculated as $\sim 9-10 \mathrm{~nm}$ for GaN grown on $\mathrm{Si}(100)$ and $\mathrm{Si}$ (111) samples while it was $\sim 5 \mathrm{~nm}$ for $\mathrm{GaN} / \mathrm{sapphire}$ sample. AFM measurements (not shown here) were performed on $\mathrm{GaN}$ deposited on $\mathrm{Si}$ (111), Si (100), and sapphire substrates. root mean square (RMS) surface roughness values were found as $0.69,0.77$, and $1.83 \mathrm{~nm}$ for $\mathrm{GaN}$ deposited on the same sequence of substrates.

\section{B. Chemical characterization}

$\mathrm{GaN}$ thin films deposited on different substrates were analyzed for elemental composition and chemical bonding states of the film from the sample surface and the bulk using XPS. Survey scans from GaN deposited on $\mathrm{Si}$ (100), Si (111), and sapphire revealed the presence of gallium, nitrogen, oxygen, and carbon with Ga 3d, N 1s, O 1s, and C 1s peaks. Survey scans from the surface of the film have been chargecorrected by referencing the $\mathrm{C} 1 \mathrm{~s}$ peak at $284.8 \mathrm{eV}$. GaN thin films were etched with $\mathrm{Ar}^{+}$ions in order to obtain XPS survey scan from the bulk of the films. The survey scans from the bulk of the film were charge-corrected by referencing the Ar $2 p$ peak at $242 \mathrm{eV}$. Elemental concentrations obtained from the surface and bulk are provided in Table I. It is evident that the very surface of GaN films was contaminated

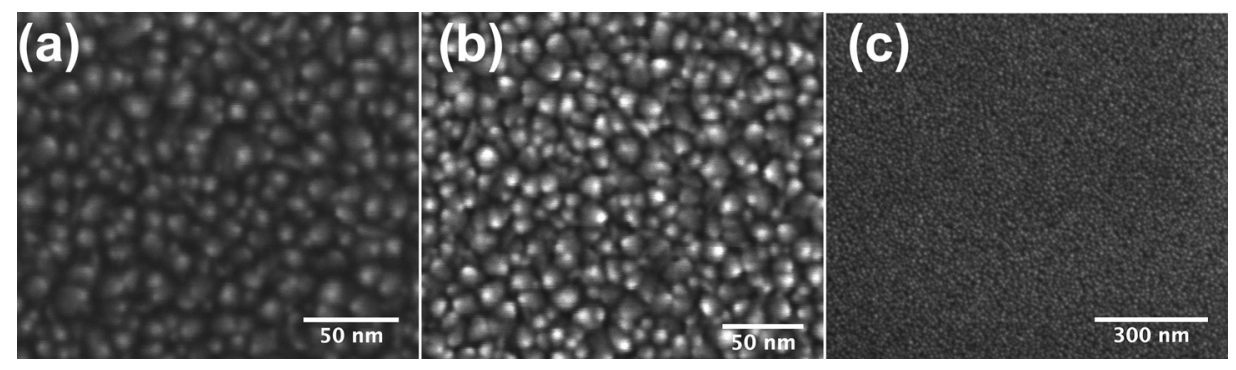

FIG. 4. SEM images of (a) GaN/Si (111), (b) GaN/Si (100), and (c) GaN/sapphire. 
TABLE I. Elemental at. \% of GaN grown on different substrates as extracted from XPS measurements.

\begin{tabular}{lcccc}
\hline \hline Substrate & C at. \% & O at. \% & Ga at. \% & N at. \% \\
\hline Sapphire (surface) & 27.94 & 12.09 & 21.91 & 38.06 \\
Sapphire (bulk) & - & 3.00 & 31.22 & 65.78 \\
Si (100) (surface) & 19.26 & 12.55 & 22.29 & 45.90 \\
Si (100) (bulk) & - & 2.98 & 31.75 & 65.27 \\
Si (111) (surface) & 20.33 & 12.69 & 23.13 & 43.86 \\
Si (111) (bulk) & - & 2.07 & 34.60 & 63.33 \\
\hline \hline
\end{tabular}

with oxygen (up to 12 at. \%) and carbon (up to 28 at. \%). Adventitious carbon and oxygen found on the surface of the film arise from postdeposition atmospheric exposure. There was no evidence of measurable carbon in the bulk of the films which shows that the surface reaction between $\mathrm{GaEt}_{3}$ and $\mathrm{N}_{2} / \mathrm{H}_{2}$ plasma led to effective removal of carbon ligands. On the other hand, there is trace of oxygen (2-3 at. \%) in the bulk of GaN films regardless of the substrate used. Probable oxygen source might include oxygen and water vapor impurities present in either plasma gases or organometallic precursors. It might also originate from trapped oxygen/water vapor inside the multilayer coatings on the inner walls of the ALD vacuum reactor. Another possibility might be the room-temperature diffusion of surface oxygen after atmospheric oxidation. Survey scans from the surface and bulk of GaN grown on Si (100), Si (111), and sapphire reveal nonstoichiometric films with higher atomic percentages for nitrogen. We have used monochromatic $\mathrm{Al} \mathrm{K}_{\alpha}$ source for XPS scans which provides better resolution. However, it also makes the analysis of survey spectra more complicated as N 1s core level peak overlaps with Ga Auger peak. ${ }^{35}$ Therefore, survey scans recorded using monochromatic Al $\mathrm{K}_{\alpha}$ source overestimate the nitrogen atomic percentage and provides inaccurate information about the stoichiometry of the film.

In order to provide a more reliable explanation for this overestimation which leads to a nearly (1:2) unrealistic stoichiometry, we decided to take into account only the relative weights of Ga-N and N-Ga peaks obtained in Ga $3 \mathrm{~d}$ and $\mathrm{N}$ 1s high resolution scans for PA-ALD and MOCVD-grown $\mathrm{GaN}$ films. This way, we omit the contributions coming from Ga Auger and Ga-Ga peaks for nitrogen and gallium concentrations, respectively. As already depicted in Table II within the manuscript, approximately $\% 39.1$ of $\mathrm{N} 1 \mathrm{~s}$ signal belongs directly to nitrogen contribution ( $\mathrm{N}-\mathrm{Ga}$ peak) for

TABLE II. Relative percentages of different bonding species present in PAALD grown GaN/Si(111) and MOCVD-grown GaN on sapphire substrates.

\begin{tabular}{lccc}
\hline \hline Sample & Peak & $\begin{array}{c}\text { Relative \% } \\
{[\text { GaN/Si(11)] }}\end{array}$ & $\begin{array}{c}\text { Relative \% } \\
\text { (MOCVD grown GaN) }\end{array}$ \\
\hline Ga 3d & Ga-N & 86.39 & 87.06 \\
& Ga-Ga & 13.61 & 12.94 \\
N 1s & N-Ga & 39.05 & 44.59 \\
& Ga-LMM & 56.58 & 51.62 \\
& Ga-LMM & 4.37 & 3.78 \\
\hline \hline
\end{tabular}

GaN/Si(111) sample, which corresponds to an effective nitrogen concentration of $24.8 \%$ when multiplied with the extracted $63.3 \%$ concentration (Table I) within the bulk of the film. Similarly, the effective Ga concentration is calculated as $29.9 \%$ after taking into account that only $86.4 \%$ of Ga $3 \mathrm{~d}$ peak contributes to the stoichiometry. Hence, the PAALD grown GaN film exhibits a relative stoichiometry of (1:0.83). In order to eliminate the preferential etching effect, we should also correct the stoichiometry via a comparison with a reference sample exhibiting ideal stoichiometry. Using the atomic concentrations given in Table I, Ga-N, and $\mathrm{N}-\mathrm{Ga}$ peak weights for MOCVD-grown sample, we obtain a relative stoichiometry of (1:0.81). As we can safely assume that commercial MOCVD-grown epitaxial GaN sample has near-ideal stoichiometry (1:1), PA-ALD-grown GaN films depicts a stoichiometry of $(1: 1.025)$, corresponding to only a slightly N-rich film with $2.5 \%$ excess nitrogen concentration with respect to epitaxial GaN film.

Figure 5 shows Ga $3 \mathrm{~d}$ and $\mathrm{N}$ 1s high resolution XPS scans from bulk of the PA-ALD-grown GaN/Si(111) and an MOCVD-grown epitaxial GaN on sapphire to investigate the individual peaks. At low energy resolution, XPS provides qualitative and quantitative information on the elements present in the material whereas high-energy resolution XPS (HR-XPS) provides information on the chemical state and bonding of those elements. Ga 3d scans [Figs. 5(a) and 5(c)] obtained from both PA-ALD and MOCVD-grown GaN samples were fitted by two subpeaks. The main subpeak reveals the existence of Ga-N bond in the bulk of the film with peak position at 19.47 and $18.97 \mathrm{eV}$ for $\mathrm{GaN} / \mathrm{Si}$ (111) and MOCVD grown GaN, respectively. ${ }^{5,35}$ Both the Ga $3 \mathrm{~d}$ spectra are dominated by $\mathrm{Ga}-\mathrm{N}$ main peak. In the literature, Ga-O component is observed at $\sim 1.2 \mathrm{eV}$ higher binding energy while Ga-Ga component was detected at $\sim 1 \mathrm{eV}$ lower binding energy compared to main $\mathrm{Ga}-\mathrm{N}$ peak binding energy. ${ }^{36,37}$ We were not able to identify any contribution from Ga-O species in the bulk of the film. Ga-Ga bonding state is observed as a second subpeak at 18.24 and $18.05 \mathrm{eV}$ in Ga 3d HR-XPS spectra of PA-ALD and MOCVD-grown $\mathrm{GaN}$, respectively. ${ }^{5,36}$ Uncoordinated $\mathrm{Ga}$ is believed to appear inside the film after Ar ion etching due to the accumulation of metallic Ga at the sample surface. ${ }^{38}$ FWHM of main Ga-N subpeak was recorded as $1.37 \mathrm{eV}$ from MOCVDgrown $\mathrm{GaN}$, while it increases to $1.79 \mathrm{eV}$ for HCPA-ALD grown GaN on Si (111). FWHM obtained from the Ga-N subpeak of MOCVD grown GaN is slightly higher than the literature reported $\mathrm{GaN}$ value of $1.1 \mathrm{eV}$, which might be ascribed to increased defect density or localized difference in charge state of the low-temperature grown ALD sample. ${ }^{30}$

$\mathrm{N}$ 1s HR-XPS spectra [Figs. 5(b) and 5(d)] were fitted using three components. First subpeak in N 1s HR-XPS spectra corresponds to N-Ga bond $(\mathrm{BE}=396.93,397.58$ for PA-ALD and MOCVD-grown GaN, respectively), ${ }^{36,39}$ and the other two subpeaks correspond to Ga-LMM auger peaks. ${ }^{35}$ The slight shift in binding energies of $\mathrm{Ga}-\mathrm{N}$ and N-Ga bonds in HCPA-ALD and MOCVD-grown GaN samples might arise due to differential charging, material defects, or nitrogen vacancies inside the ALD-grown GaN 

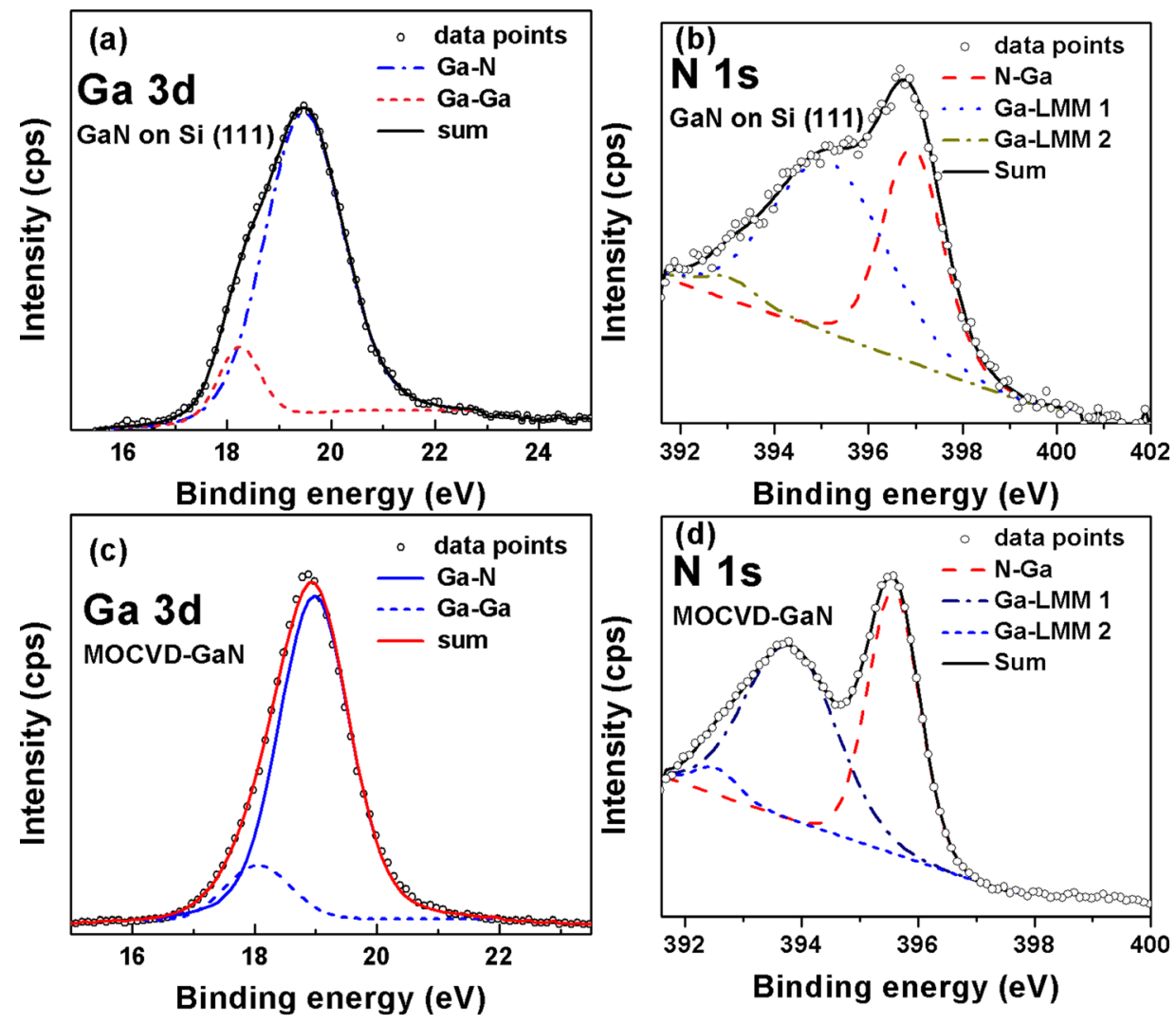

FIG. 5. (Color online) High-resolution XPS scans of Ga 3d and N 1s obtained from bulk of GaN layers grown [(a) and (b)] by low-temperature PA-ALD on Si (111) and [(c) and (d)] by high-temperature MOCVD on sapphire substrate.

film. FWHM of main N-Ga peak was recorded as 1.06 and $1.53 \mathrm{eV}$ for MOCVD grown GaN and HCPA-ALD grown $\mathrm{GaN}$, respectively. FWHM of $\mathrm{N}-\mathrm{Ga}$ peak for MOCVD grown $\mathrm{GaN}$ is slightly higher than the literature reported value $(0.85 \mathrm{eV})$ for bulk GaN. Relative percentages of different bonding species were calculated for both samples based on the area under the corresponding peaks and are provided in Table II. Relative percentages of different bonding species in GaN/Si (111) and MOCVD-grown GaN are close to each other which indicates that stoichiometry of GaN grown on $\mathrm{Si}$ (111) resembles with epitaxial GaN sample, confirming the

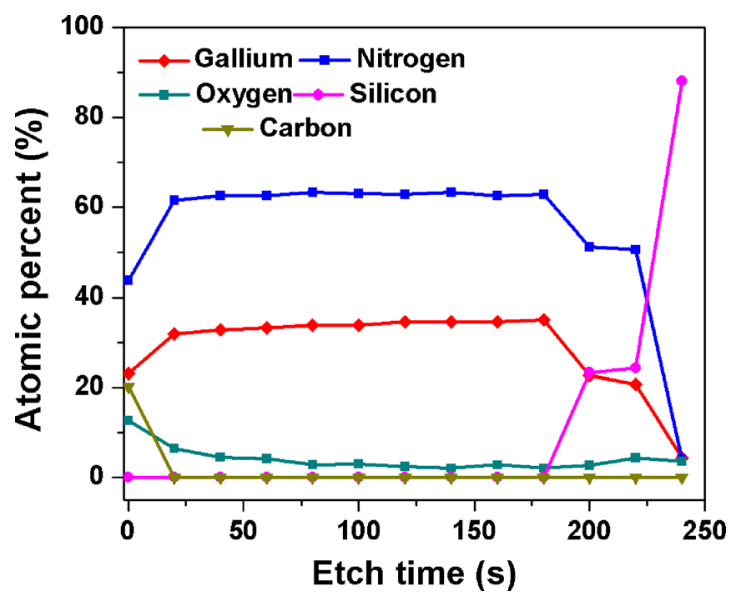

FIG. 6. (Color online) Depth profile experiment performed on Ga/Si (111) and the thickness evolution of the related elemental concentrations. stoichiometry calculation based on the Ga-N and N-Ga subpeaks carried out above. ${ }^{35}$ Peak positions of Ga $3 \mathrm{~d}$ and $\mathrm{N} 1 \mathrm{~s}$ HR-scans (not shown here) obtained from bulk of $\mathrm{GaN}$ grown on $\mathrm{Si}(100)$ and sapphire matches well with the scans obtained from bulk of GaN grown on Si (111).In Fig. 6, $\mathrm{GaN} / \mathrm{Si}(111)$ depth profile analysis is provided, which indicates the elemental composition variation between GaN-air and GaN-Si interface. Results reveal that atomic percentages of gallium and nitrogen remained fairly constant along the etching direction throughout the film thickness. Carbon disappears after the very first etching cycle while oxygen stabilizes to around $\sim 2-3$ at. $\%$ in the bulk of the film.

\section{SUMMARY AND CONCLUSION}

We have presented our efforts for understanding the substrate effect on GaN thin films grown using low-temperature HCPA-ALD. GaN thin films were deposited on Si (111), Si (100), and c-plane sapphire substrates at a growth temperature of $200{ }^{\circ} \mathrm{C}$ using $\mathrm{GaEt}_{3}$ precursor and $\mathrm{N}_{2} / \mathrm{H}_{2}$ plasma as $\mathrm{Ga}$ and nitrogen source, respectively. Unlike high-temperature heteroepitaxy, neither lattice mismatch nor thermal expansion coefficient showed significant importance for low-temperature HCPA-ALD grown GaN. XRR measurements showed film density values of $5.70,5.74$, and $5.54 \mathrm{~g} / \mathrm{cm}^{3}$ for GaN grown on Si (100), Si (111), and sapphire, respectively. All HCPAALD grown GaN samples showed hexagonal polycrystalline wurtzite structure. Dominant GIXRD diffraction peaks for GaN films grown on $\mathrm{Si}$ and sapphire substrates were detected 
as (002) and (103), respectively. A better crystalline quality and higher film density obtained from GaN grown on Si (111) is attributed to the hexagonal nature of Si (111) substrate surface. Grain size of the films was determined to be $\sim 9-10 \mathrm{~nm}$ for GaN grown on Si (100) and Si (111) samples while it was $\sim 5 \mathrm{~nm}$ for $\mathrm{GaN} /$ sapphire sample. RMS surface roughness values were found as $0.68,0.76$, and $1.83 \mathrm{~nm}$ for $\mathrm{GaN}$ deposited on Si (111), Si (100), and sapphire. All samples exhibited similar refractive index values $(\sim 2.17$ at $632 \mathrm{~nm})$ with $2 \%-3 \%$ of oxygen impurity existing within the bulk of the films and with no detectable carbon incorporation. Optical band gap values of 3.44, 3.45, and $3.61 \mathrm{eV}$ were recorded for GaN grown on Si (100), Si (111), and sapphire substrates, respectively. Higher growth rate observed for GaN grown on sapphire was attributed to a more-effective nitridation and faster nucleation process on the sapphire surface.

${ }^{1}$ P. F. Yang, S. R. Jian, Y. S. Lai, C. S. Yang, and R. S. Chen, J. Alloys Compd. 463, 533 (2008).

${ }^{2}$ A. Haider, S. Kizir, C. Ozgit-Akgun, E. Goldenberg, S. A. Leghari, A. K. Okyay, and N. Biyikli, J. Mater. Chem. C 3, 9620 (2015).

${ }^{3}$ H. Na, S. Takado, S. Sawada, M. Kurouchi, T. Akagi, H. Naoi, T. Araki, and Y. Nanishi, J. Cryst. Growth 300, 177 (2007).

${ }^{4}$ X. Gao, C. Liu, C. Yin, L. Sun, D. Tao, C. Yang, and B. Man, J. Magn. Magn. Mater. 343, 65 (2013).

${ }^{5}$ C. Ozgit-Akgun, E. Goldenberg, A. K. Okyay, and N. Biyikli, J. Mater. Chem. C 2, 2123 (2014).

${ }^{6}$ T. Wang, J. Bai, and S. Sakai, J. Cryst. Growth 224, 5 (2001).

${ }^{7}$ H. Y. Chung, K. Y. Woo, S. J. Kim, and T. G. Kim, Opt. Commun. 331, 282 (2014).

${ }^{8}$ M. L. Lee, J. K. Sheu, Y. K. Su, S. J. Chang, W. C. Lai, and G. C. Chi, IEEE Electron Device Lett. 25, 593 (2004).

${ }^{9}$ A. El Fatimy et al., J. Appl. Phys. 107, 2 (2010).

${ }^{10}$ R. Dimitrov, A. Mitchell, L. Wittmer, O. Ambacher, M. Stutzmann, J. Hilsenbeck, and W. Rieger, Jpn. J. Appl. Phys., Part 1 38, 4962 (1999).

${ }^{11}$ J. N. Dai et al., J. Electron. Mater. 38, 1938 (2009).

${ }^{12}$ T.-Y. Tsai, S.-L. Ou, M.-T. Hung, D.-S. Wuu, and R.-H. Horng, J. Electrochem. Soc. 158, H1172 (2011).

${ }^{13}$ B. L. Liu, M. Lachab, A. Jia, A. Yoshikawaa, and K. Takahashi, J. Cryst. Growth 234, 637 (2002).
${ }^{14}$ D. Doppalapudi, E. Iliopoulos, S. N. Basu, and T. D. Moustakas, J. Appl. Phys. 85, 3582 (1999).

${ }^{15}$ S. A. Kukushkin, A. V. Osipov, V. N. Bessolov, B. K. Medvedev, V. K. Nevolin, and K. A. Tcarik, Rev. Adv. Mater. 17, 1 (2008).

${ }^{16}$ D. Zhu, D. J. Wallis, and C. J. Humphreys, Rep. Prog. Phys. 76, 106501 (2013).

${ }^{17}$ S. Pal and C. Jacob, Bull. Mater. Sci. 27, 501 (2004).

${ }^{18}$ C.-A. Chang et al., Jpn. J. Appl. Phys., Part 145,2516 (2006).

${ }^{19}$ E. Arslan, M. K. Ozturk, A. Teke, S. Ozcelik, and E. Ozbay, J. Phys. D: Appl. Phys. 41, 155317 (2008).

${ }^{20}$ R. L. Puurunen, J. Appl. Phys. 97, 121301 (2005).

${ }^{21}$ H. B. Profijt, S. E. Potts, M. C. M. van de Sanden, and W. M. M. Kessels, J. Vac. Sci. Technol., A 29, 050801 (2011).

${ }^{22}$ K. E. C. M. Krishnan, R. G. Jahn, and W. F. von Jaskowsky, AIAA J. 15, 1217 (1977).

${ }^{23}$ P. Motamedi and K. Cadien, RSC Adv. 5, 57865 (2015).

${ }^{24}$ P. Motamedi, N. Dalili, and K. C. Cadien, J. Mater. Chem. C 3, 7428 (2015).

${ }^{25}$ M. Alevli, N. Gungor, A. Haider, S. Kizir, S. A. Leghari, and N. Biyikli, J. Vac. Sci. Technol., A 34, 01A125 (2016).

${ }^{26}$ M. Alevli, A. Haider, S. Kizir, S. A. Leghari, and N. Biyikli, J. Vac. Sci. Technol., A 34, $01 \mathrm{~A} 137$ (2016).

${ }^{27}$ S. Banerjee, A. A. I. Aarnink, R. Van de Kruijs, A. Y. Kovalgin, and J. Schmitz, Phys. Status Solidi 12, 1036 (2015).

${ }^{28}$ K. S. A. Butcher, D. Alexandrov, P. Terziyska, V. Georgiev, D. Georgieva, and P. W. Binsted, Phys. Status Solidi 209, 41 (2012).

${ }^{29}$ O. H. Kim, D. Kim, and T. J. Anderson, J. Vac. Sci. Technol., A 27, 923 (2009).

${ }^{30}$ N. Nepal, V. R. Anderson, J. K. Hite, and C. R. Eddy, Thin Solid Films 589, 47 (2015).

${ }^{31}$ E. Rosencher, Optoelectronics (Cambridge University, 2002), p. 304.

${ }^{32}$ C. Liu, S. Stepanov, A. Gott, P. A. Shields, E. Zhirnov, W. N. Wang, E. Steimetz, and J. T. Zettler, Phys. Status Solidi C 3, 1884 (2006).

${ }^{33}$ M. Yasaka, Rigaku J. 26, 1 (2010).

${ }^{34}$ S. Strite, J. Vac. Sci. Technol., B 10, 1237 (1992).

${ }^{35}$ S. S. Kushvaha, M. S. Kumar, A. K. Shukla, B. S. Yadav, D. K. Singh, M. Jewariya, S. R. Ragam, and K. K. Maurya, RSC Adv. 5, 87818 (2015).

${ }^{36}$ D. Li, M. Sumiya, S. Fuke, D. Yang, D. Que, Y. Suzuki, and Y. Fukuda, J. Appl. Phys. 90, 4219 (2001).

${ }^{37}$ M. Petravic, V. A. Coleman, K.-J. Kim, B. Kim, and G. Li, J. Vac. Sci. Technol., A 23, 1340 (2005).

${ }^{38}$ K. S. A. Butcher, Afifuddin, T. L. Tansley, N. Brack, P. J. Pigram, H. Timmers, K. E. Prince, and R. G. Elliman, Appl. Surf. Sci. 230, 18 (2004).

${ }^{39}$ M. R. Coan, J. H. Woo, D. Johnson, I. R. Gatabi, and H. R. Harris, J. Appl. Phys. 112, 4 (2012). 